\title{
A REFORMULATION OF $S$ AND $L$
}

\author{
JUDY ROITMAN ${ }^{1}$
}

\begin{abstract}
We give a combinatorial statement which is equivalent to the existence of an $S$ space, and one equivalent to the existence of an $L$ space, thus verifying the widespread intuition that the problem of $S$ and $L$ is essentially set-theoretic.
\end{abstract}

0 . Introduction. An $S$ space is regular hereditarily separable non-Lindelöf; an $L$ space is regular hereditarily Lindelöf nonseparable. The open question is whether there are real $S$ or $L$ spaces. More consistency results are known than we can list here. Important for our motivation, however, is the early anonymous observation that a Suslin line is an $L$ space, and M. E. Rudin's construction of the first known $S$ space from a Suslin tree ( = a tree of height $\omega_{1}$ with no uncountable antichains). Since an early result of Miller is that a Suslin line exists iff a Suslin tree does, it was reasonable to ask if the existence of $S$ or $L$ spaces implied the existence of Suslin trees. From [H-J] we learn that this is not the case. Nevertheless we can try to get as close as possible to such a conclusion. Here we define tangles, and show that the existence of $S$ or $L$ spaces is equivalent to the existence of appropriate sets of tangles with, roughly speaking, no uncountable antichains.

1. Statement. For convenience, we define a graph $G$ as a subset of $\left[\omega_{1}\right]^{2} \cup \omega_{1}$. We say that $G$ has height $\omega_{1}$ if no $\omega_{1}-\alpha$ is homogeneous, $\alpha<\omega_{1}$. We remind the reader that $[A]^{k}$ is the set of subsets of $A$ with cardinality $k$.

Let $G$ be a graph. We define, for $n \in \omega$, the upper $n$-tangle for $G, T^{n, G}$, and the lower $n$-tangle for $G, T_{n, G}$, as follows:

Let $a, b \in\left[\omega_{1}\right]^{n+1}$. We say that $a, b$ are separated if $a=\left\{\alpha_{0}, \ldots, \alpha_{n}\right\}, b=$ $\left\{\beta_{0}, \ldots, \beta_{n}\right\}$ where $\alpha_{j}<\alpha_{j+1}, \beta_{j}<\beta_{j+1}$, and $\alpha_{n}<\beta_{0}$. For separated $a, b \in$ $\left[\omega_{1}\right]^{n+1}$, we say

$$
a \stackrel{k}{\sim} b \text { iff }\left[j \geqslant k \Rightarrow\left(\left\{\alpha_{k}, \beta_{j}\right\} \in G \text { iff }\left\{\beta_{k}, \beta_{j}\right\} \in G\right) ;\right.
$$

$$
\text { and } \left.j<k \Rightarrow\left\{\alpha_{k}, \alpha_{j}\right\} \notin G\right]
$$

and we say

Received by the editors November 30, 1976 and, in revised form, January 5, 1977.

AMS (MOS) subject classifications (1970). Primary 54G20; Secondary 54A25, 54D20.

Key words and phrases. $S$ space, $L$ space, graph.

${ }^{1}$ The author was partially supported during the writing of this paper by NSF contract \#MCS 7610430. 


$$
\begin{aligned}
& \underset{k}{a \sim} b \text { iff }\left[j \leqslant k \Rightarrow\left(\left\{\beta_{k}, \alpha_{j}\right\} \in G \text { iff }\left\{\alpha_{k}, \alpha_{j}\right\} \in G\right)\right. \\
& \text { and } \left.j>k \Rightarrow\left\{\beta_{k}, \alpha_{j}\right\} \notin G\right] .
\end{aligned}
$$

Then $T^{n, G}$ is $\left[\omega_{1}\right]^{n+1}$ under the relations $\sim^{k}$ for $k \leqslant n$; while $T_{n, G}$ is $\left[\omega_{1}\right]^{n+1}$ under the relations $\sim_{k}$ for $k \leqslant n$. We say that $a$ and $b$ are upper $k$-compatible if $a \sim^{k} b$; lower $k$-compatible if $a \sim_{k} b$. Where the context is clear we just speak of $k$-compatibility. Thus if $T$ is either an upper or lower $n$-tangle for $G$, we define a $k$-antichain in $T$ as a subset of $T$ whose every pair is separated, but with no pair $k$-compatible.

MaIn Proposition. $\exists$ an $S$ space iff $\exists$ a graph $G$ of height $\omega_{1}$ such that no upper $n$-tangle for $G$ has an uncountable $k$-antichain for $k \leqslant n$; similarly, $\exists$ an $L$ space iff $\exists$ a graph $G$ of height $\omega_{1}$ such that no lower $n$-tangle for $G$ has an uncountable $k$-antichain for $k \leqslant n$.

2. Proof. The proof proceeds by a series of observations, none of which are profound, some of which are quite old, and few of which are published. Since many are not obvious to some one who has not spent a lot of time on $S$ and $L$, this is as good a place as any to make esoteric secrets public. Uncredited claims mentioned up to and including Observation 9 are either folklore or should be.

Define a space $X$ as right-separated iff $X=\left\{x_{\alpha}: \alpha<\omega_{1}\right\}$ and every initial segment is open; $X=\left\{x_{\alpha}: \alpha<\omega_{1}\right\}$ is left-separated iff every terminal segment is open. Note that a right-separated space is not Lindelöf, and a left-separated space is not separable.

ObSERVATION 1 (JUHASZ-HAJNAL). $X$ is hereditarily separable iff it has no left-separated subspace. $X$ is hereditarily Lindelöff iff it has no right-separated subspace.

Proof. Can be found in $[\mathbf{J}]$.

Corollary 2 (JuHASz-HAJNAL). Every $S$ space contains one which is rightseparated; every L space contains one which is left-separated.

Corollary 3 (Juhasz-HaJnal). A right-separated space is an $S$ space iff it contains no uncountable discrete subspace; a left-separated space is an L space iff it contains no uncountable discrete subspace.

ObSERvation 4. (a) (Juhasz-Hajnal). A regular right-separated space is 0-dimensional.

(b) (Kunen). $\neg \mathrm{CH} \Rightarrow$ a regular Lindelöf space of cardinality $\omega_{1}$ is 0 -dimensional.

Proof. In either case, the spaces in question are completely regular-in (a) because there is a basis of countable neighborhoods; in (b) because regular + Lindelöf $\Rightarrow$ normal. Let $X$ be a space satisfying either the hypothesis of (a) or of (b). Given a point $x \in u$, where $u$ is a basic neighborhood-countable in case (a)-let $f: X \rightarrow \mathbf{R}$ separate $x$ and $X-u$. Pick $r \in \mathbf{R}, f^{-1}(r)=\varnothing$. Then $f^{-1}([0, r])$ is the clopen neighborhood refining $u$ which we want. 
COROllaRy 5. $\exists$ an $S$ space iff $\exists$ a 0 -dimensional right-separated one. $\exists$ an $L$ space iff $\exists a$ 0-dimensional left-separated one.

Proof. From Observation 4, Corollary 2, and the theorem of Juhasz and Hajnal that $\mathrm{CH} \Rightarrow \exists$ a 0 -dimensional $L$ space.

COROLlARY 6. $\exists$ an $S$ space iff $\exists a$ Hausdorff $S$ space. $\exists$ an L space iff $\exists a$ Hausdorff $L$ space.

Proof. Given a 0 -dimensional $S$ or $L$ space $X$ of cardinality $\omega_{1}$ with basis $\mathcal{U}$, identify $X$ as a set with a subset of ${ }^{\omega} \omega$. Then the topology given by $\left\{u \cap v: u \in \mathcal{Q}, v\right.$ basic for $\left.{ }^{\omega} \omega\right\}$ is still a 0 -dimensional $S$ or $L$ space.

Thus the question for Hausdorff spaces is the same as for all spaces, and the usual assumption that all interesting spaces are Hausdorff need not be made.

OBSERVATION 7. $\exists$ an $S$ space (respectively, an $L$ space) iff $\exists$ a right-separated (respectively, left-separated) 0-dimensional one with a basis of cardinality $\omega_{1}$.

Proof. Given a 0 -dimensional right-separated $S$ or left-separated $L$ space, construct a weaker 0-dimensional topology in $\omega_{1}$ steps that remains, respectively, right- or left-separated. This is still an $S$ or $L$ space.

Before the next observation, if $X=\left\{x_{\alpha}: \alpha<\omega_{1}\right\} \subset{ }^{\omega_{1}} 2$, we define its dual $X^{*}=\left\{y_{\alpha}: \alpha<\omega_{1}\right\}$ where $y_{\alpha}(\beta)=x_{\beta}(\alpha)$. We say $X$ has countable support iff each $x_{\alpha} \in X$ does.

OBSERVATION 8. $\exists$ an $S$ space iff $\exists$ one which is a subspace of $\omega_{12}$ and whose dual has countable support. $\exists$ an $L$ space iff $\exists$ one which is a subspace of $\omega_{1} / 2$ with countable support.

Proof. Given the spaces guaranteed by Observation 7, we code each one as a subset of $\omega_{1} 2$ by listing a clopen collection $\left\{u_{\alpha}: \alpha<\omega_{1}\right\}$, guaranteeing for the $S$ space that each $u_{\alpha} \subset\left\{x_{\beta}: \beta \leqslant \alpha\right\}$, and for the $L$ space that each $u_{\alpha} \cap\left\{x_{\beta}: \beta \leqslant \alpha\right\}=\varnothing$.

OBSERVATION 9. $\exists$ an $S$ space iff $\exists$ an $S$ space $Y=\left\{y_{\alpha}: \alpha<\omega_{1}\right\} \subset{ }^{\omega_{1}} 2$ where supp $y_{\alpha} \cap \alpha=\varnothing$. $\exists$ an $L$ space iff $\exists$ an $L$ space $Y=\left\{y_{\alpha}: \alpha<\omega_{1}\right\}$ $\subset{ }^{\omega_{1}} 2$ where supp $y_{\alpha} \subset \alpha+1$. (Note. By Observation 6 the $y_{\alpha}$ need not be distinct.)

Proof. Given $X$ as in Observation 8, pick uncountable $Y \subset X$ such that, for the $S$ space, $\alpha<\beta$ and $x_{\alpha}, x_{\beta} \in Y \Rightarrow \inf \left(\operatorname{supp} x_{\alpha}\right)<\inf \left(\operatorname{supp} x_{\beta}\right)$; and for the $L$ space, $\alpha<\beta$ and $x_{\alpha}, x_{\beta} \in Y \Rightarrow \sup \left(\operatorname{supp} x_{\alpha}\right)<\sup \left(\operatorname{supp} x_{\beta}\right.$ ). Reindex the points of $Y$ by a set $A \subset \omega_{1}$ so that $\gamma \in A, y_{\gamma} \in Y \Rightarrow \gamma=$ $\inf \left(\operatorname{supp} y_{\gamma}\right.$ ) for the $S$ space, $\gamma+1=\sup \left(\operatorname{supp} y_{\gamma}\right.$ ) for the $L$ space. If $\gamma \notin S$, let $y_{\gamma}$ be 0 everywhere.

Observation 9 gives us the canonical spaces we will need.

Now suppose $X=\left\{x_{\alpha}: \alpha<\omega_{1}\right\} \subset{ }^{\omega_{1}} 2$, and we want to know whether it has an uncountable discrete subspace-by Corollary 3 and Observation 8 this is all we need to know. Given an uncountable $A \subset \omega_{1}$, define $X_{A}=\left\{x_{\alpha} \in X\right.$ : $\alpha \in A\}$. Suppose $\mathscr{Q}=\left\{u_{\alpha}: \alpha \in A, x_{\alpha} \in u_{\alpha}\right\}$ is a basic clopen cover of $X_{A}$. 
We want to know whether $\mathscr{U}$ is a discrete cover of $X_{A}$, i.e. whether each $u_{\alpha} \cap X_{A}=\left\{x_{\alpha}\right\}$. W.l.o.g. we may assume that $u_{\alpha} \subset\left\{x: x(\alpha)=x_{\alpha}(\alpha)\right\}$. Then $\left\langle X_{A}, \mathscr{Q}\right\rangle$ can be coded as a subset of $\omega \times\left[\omega_{1} \times 2\right]<\omega$ where the code for $\left\langle x_{\alpha}, u_{\alpha}\right\rangle$ is $\left\langle k_{\alpha},\left\langle\delta_{\alpha}^{0}, i_{\alpha}^{0}\right\rangle, \ldots,\left\langle\delta_{\alpha}^{n_{\alpha}}, i_{\alpha}^{n_{\alpha}}\right\rangle\right\rangle$ and $k_{\alpha} \leqslant n_{\alpha}, \alpha=\delta_{\alpha}^{k_{\alpha}}, u_{\alpha}=\{x$ : $\left.x\left(\delta_{\alpha}^{j}\right)=i_{\alpha}^{j}, j \leqslant n_{\alpha}\right\}$. Since $X_{A}$ is discrete under $\mathscr{U} \Rightarrow X_{B}$ is discrete under Q $\uparrow B=\left\{u_{\alpha}: \alpha \in B\right\}$ for all $B \subset A$, by a counting argument we may assume w.l.o.g. that $\alpha, \beta \in A \Rightarrow k_{\alpha}=k_{\beta}=k, n_{\alpha}=n_{\beta}=n$; we say that the set of codes for $\left\langle X_{A}, \mathcal{Q}\right\rangle$ is uniform. By the usual $\Delta$-system argument we may further w.l.o.g. assume that $\alpha, \beta \in A, \alpha<\beta \Rightarrow \delta_{\alpha}^{n}<\delta_{\beta}^{0}$; we say that the set of codes for $\left\langle X_{A}, \mathscr{Q}\right\rangle$ is separated. Given a uniform, separated set of codes for $\left\langle X_{A}, \mathscr{Q}\right\rangle$, we further note that

$$
\alpha, \beta \in A \Rightarrow\left[x_{\alpha} \in u_{\beta} \text { iff } \forall j \leqslant n\left(x_{\alpha}\left(\delta_{\beta}^{j}\right)=i_{\beta}^{j}=x_{\beta}\left(\delta_{\beta}^{j}\right)\right)\right]
$$

that $\forall \alpha \in A\left[\operatorname{supp} x_{\alpha} \cap \alpha=\varnothing\right] \Rightarrow$ each $i_{\alpha}^{j}=0$ for $j<k$; while $\forall \alpha \in$ $A\left[\operatorname{supp} x_{\alpha} \subset \alpha+1\right]$ each $i_{\alpha}^{j}=0$ for $j>k$. We are inexorably led into tangles.

Definition 10. Let $X=\left\{x_{\alpha}: \alpha<\omega_{1}\right\} \subset{ }^{\omega_{1}} 2$. Then the right graph for $X$,

$$
G^{X}=\left\{\{\alpha, \beta\}: \alpha \leqslant \beta \text { and } x_{\alpha}(\beta)=1\right\} ;
$$

the left graph for $X$,

$$
G_{X}=\left\{\{\alpha, \beta\}: \alpha \geqslant \beta \text { and } x_{\alpha}(\beta)=1\right\} .
$$

Definition 11. Given a graph $G$ we define its right space, $X^{G} \subset{ }^{\omega_{1}} 2$, by

$$
X^{G}=\left\{x_{\alpha}: x_{\alpha}(\beta)=1 \text { iff } \alpha \leqslant \beta \text { and }\{\alpha, \beta\} \in G\right\} .
$$

We define the left space of $G, X_{G} \subset{ }^{\omega_{1}} 2$, by

$$
X_{G}=\left\{x_{\alpha}: x_{\alpha}(\beta)=1 \text { iff } \alpha \geqslant \beta \text { and }\{\alpha, \beta\} \in G\right\} .
$$

Note that for $X=\left\{x_{\alpha}: \alpha<\omega_{1}\right\} \subset{ }^{\omega_{1}} 2$, if each supp $x_{\alpha} \cap \alpha=\varnothing$ then $X=X^{\left(G^{x}\right)}$; while if each supp $x_{\alpha} \subset \alpha+1$ then $X=X_{\left(G_{X}\right)}$. On the other hand, given a graph $G$, supp $x_{\alpha} \cap \alpha=\varnothing$ for each $x_{\alpha} \in X^{G}$, and supp $x_{\alpha} \subset \alpha$ +1 for each $x_{\alpha} \in X_{G}$; also $G^{\left(X^{G}\right)}=G_{\left(X_{G}\right)}=G$. Thus, by Observation 9, looking at graphs is the same as looking at spaces. $X^{G}$ is, of course, the dual of $X_{G}$, and it is of interest to note that, by a modification of the spaces in [H-J], $X^{G}$ may be $S$ without $X_{G}$ being $L$ and vice versa.

OBSERVATION 12. If $G$ does not have height $\omega_{1}$ then $X^{G}$ is either Lindelöf or not hereditarily separable, and $X_{G}$ is either separable or not hereditarily Lindelöf.

Proof. If some $\omega_{1}-\alpha$ is homogeneous for 0 , then $X^{G}$ and $X_{G}$ are homeomorphic to subsets of ${ }^{\omega} \omega$. If some $\omega_{1}-\alpha$ is homogeneous for 1 , then $\left\{x_{\beta}: \beta>\alpha\right\}$ is a discrete subspace of, respectively, $X^{G}$ or $X_{G}$.

We are now ready to prove the main proposition, doing it for $S$ spaces and letting the reader make the obvious changes for $L$ spaces.

Suppose $X=\left\{x_{\alpha}: \alpha<\omega_{1}\right\} \subset^{\omega_{1}} 2$ is an $S$ space and supp $x_{\alpha} \cap \alpha=\varnothing$. By Observation $12, G^{X}$ has height $\omega_{1}$. We want to prove that $\forall n \forall k \leqslant n\left(T^{n, G^{X}}\right.$ has no uncountable $k$-antichains). On the other hand, given a graph $G$ of 
height $\omega_{1}$ such that $\forall n \forall k \leqslant n\left(T^{n, G}\right.$ has no uncountable $k$-antichains), we want to prove that $X^{G}$ is an $S$ space.

By the discussion after Definition 11, we can prove both directions simultaneously, letting $X=X^{G}$ where $G$ has height $\omega_{1}$. We shall prove that $S$ is an $S$ space iff $G$ has the required property.

Let $\left\langle X_{A}, \mathscr{Q}\right\rangle$ have a uniform, separated set of codes. If $a^{*}=\langle k$, $\left.\left\langle\delta_{\alpha}^{0}, i_{\alpha}^{0}\right\rangle, \ldots,\left\langle\delta_{\alpha}^{n}, i_{\alpha}^{n}\right\rangle\right\rangle$ is such a code, let $a_{\alpha}=\left\langle\delta_{\alpha}^{0}, \ldots, \delta_{\alpha}^{n}\right\rangle$. Then by the discussion prior to Definition 10, if $\alpha, \beta \in A$ then

$$
\begin{aligned}
x_{\alpha} \in u_{\beta} \text { iff }\left[j \geqslant k \Rightarrow\left(\left\{\delta_{\alpha}^{k}, \delta_{\beta}^{j}\right\} \in G\right.\right. & \text { iff } \left.\left\{\delta_{\beta}^{k}, \delta_{\beta}^{j}\right\} \in G\right) ; \\
& \text { and } \left.j<k \Rightarrow\left\{\delta_{\alpha}^{k}, \delta_{\beta}^{j}\right\} \notin G\right],
\end{aligned}
$$

i.e. $x_{\alpha} \in U_{\beta}$ iff $a_{\alpha} \sim^{k} a_{\beta}$ in $T^{n, G}$. Thus $\mathscr{U}$ is a discrete cover of $X_{A}$ iff $\left\{a_{\alpha}\right.$ : $\alpha \in A\}$ is a $k$-antichain in $T^{n, G}$. On the other hand, given a $k$-antichain $Z$ in $T^{n, G}$, for $a=\left\{\delta_{a}^{0}, \ldots, \delta_{a}^{n}\right\} \in Z$, define $a^{*}=\left\langle k,\left\langle\delta_{a}^{0}, i_{a}^{0}\right\rangle, \ldots,\left\langle\delta_{a}^{n}, i_{a}^{n}\right\rangle\right\rangle$ where $i_{a}^{j}=1$ iff $j \geqslant k$ and $\left\{\delta_{a}^{i}, \delta_{a}^{j}\right\} \in G$. Then $\left\{a^{*}: a \in Z\right\}$ is a set of codes for $\left\langle X_{A}, \mathscr{U}\right\rangle$ where $\mathcal{U}$ is a discrete cover of $X_{A}, A=\left\{\delta_{a}^{k}: a \in Z\right\}$.

Since $X$ is an $S$ space iff $X$ has no uncountable discrete subsets, we finally conclude that $X$ is an $S$ space iff $\forall n \forall k \leqslant n$ ( $T^{n, G}$ has no uncountable $k$-antichains).

\section{BIBLIOGRAPHY}

[H-J] A. Hajnal and I. Juhasz, On hereditarily $\alpha$-Lindelöf and hereditarily $\alpha$-separable spaces. II, Fund. Math. 81 (1973/74), 147-158.

[J] I. Juhasz, Cardinal functions in topology, Math. Centre Tracts, no. 34, Mathematisch Centrum, Amsterdam, 1971.

[M] E. W. Miller, A note on Souslin's problem, Amer. J. Math. 65 (1943), 673-678.

[R] M. E. Rudin, A normal hereditarily separable non-Lindelöf space, Illinois J. Math. 16 (1972), 621-626.

Department of Mathematics, Wellesley College, Wellesley, Massachusetts 01281

Current address: Department of Mathematics, University of Kansas, Lawrence, Kansas 66044 